\title{
Genome-wide phylogenetic analysis of the pathogenic potential of Vibrio furnissii
}

\author{
Thomas M. Lux, Rob Lee and John Love* \\ Biosciences, College of Life and Environmental Sciences, The University of Exeter, Exeter, UK
}

\section{Edited by:}

Hongyue Dang, Xiamen University, China

Reviewed by:

Li Sun, Chinese Academy of

Sciences, China

Roberto Bastías, Pontifical Catholic

University of Valparaiso, Chile

*Correspondence:

John Love, Biosciences, College of

Life and Environmental Sciences,

The Geoffrey Pope Building, The

University of Exeter, Stocker Road,

Exeter EX4 4QD, UK

e-mail: j.love@exeter.ac.uk
We recently reported the genome sequence of a free-living strain of Vibrio furnissii (NCTC 11218) harvested from an estuarine environment. V. furnissii is a widespread, free-living proteobacterium and emerging pathogen that can cause acute gastroenteritis in humans and lethal zoonoses in aquatic invertebrates, including farmed crustaceans and molluscs. Here we present the analyses to assess the potential pathogenic impact of $V$. furnissii. We compared the complete genome of $V$. furnissii with 8 other emerging and pathogenic Vibrio species. We selected and analyzed more deeply 10 genomic regions based upon unique or common features, and used 3 of these regions to construct a phylogenetic tree. Thus, we positioned $V$. furnissii more accurately than before and revealed a closer relationship between $V$. furnissii and $V$. cholerae than previously thought. However, $V$. furnissii lacks several important features normally associated with virulence in the human pathogens $V$. cholera and $V$. vulnificus. A striking feature of the $V$. furnissii genome is the hugely increased Super Integron, compared to the other Vibrio. Analyses of predicted genomic islands resulted in the discovery of a protein sequence that is present only in Vibrio associated with diseases in aquatic animals. We also discovered evidence of high levels horizontal gene transfer in $V$. furnissii. $V$. furnissii seems therefore to have a dynamic and fluid genome that could quickly adapt to environmental perturbation or increase its pathogenicity. Taken together, these analyses confirm the potential of $V$. furnissii as an emerging marine and possible human pathogen, especially in the developing, tropical, coastal regions that are most at risk from climate change.

Keywords: Vibrio furnissii, horizontal gene transfer, genome comparison, emerging pathogens, pathogenicity islands, phylogenetic analysis, genome phylogeny

\section{INTRODUCTION}

The Vibrionales are a diverse order of free-living, gram-negative proteobacteria, found throughout the world in marine and freshwater environments (Baumann et al., 1983; Thompson et al., 2004). Vibrio cholerae, V. parahaemolyticus and V. vulnificus are potentially lethal human pathogens (Baumann et al., 1983; Chakraborty et al., 1997; Rivera et al., 2001; Colwell, 2004; Thompson et al., 2004) and at least eight other Vibrio species pose a serious threat to human health either through ingestion of contaminated food-notably raw seafood-or by exposure of skin lesions to aquatic environments and marine animals. These "emerging Vibrio" include Vibrio furnissii, a widespread, marine species (Hickman-Brenner et al., 1984). V. furnissii is considered a relatively weak human pathogen, although is has been implicated in occasional outbreaks of acute gastroenteritis in which deaths have been recorded (Chakraborty et al., 1997; Dalsgaard et al., 1997; Wu et al., 2007). More importantly, V. furnissii has caused zoonoses in marine invertebrates (Matté et al., 1994; Austin, 2010) as significant as those caused by other species such as $V$. corallyticus (Ben-Haim et al., 2003) and V. splendidus (Gay et al., 2004). It is therefore possible that $V$. furnissii could present an increasing risk to human economic activity, notably the production of farmed shellfish in developing countries.
The V. furnissii genome (Strain NCTC 11218) (HickmanBrenner et al., 1984) was completely sequenced, assembled and annotated (Lux et al., 2011). In the present analysis, we have assessed the pathogenic potential of Vibrio furnissii. Individual phylogenetic trees for all predicted gene products were constructed and showed a closer evolutionary relationship between $V$. furnissii and V. cholerae than hitherto recognized. An identification of putative pathogenicity islands in 8 sequenced Vibrionaceae genomes and analysis of their distribution revealed stark congruities. Moreover, we noted a high abundance of horizontal gene transfer in the $V$. furnissii genome from other marine bacteria. Taken together, these analyses confirm the potential of $V$. furnissii as an emerging marine and possible human pathogen.

\section{MATERIALS AND METHODS} REPRESENTATION AND ANALYSIS OF THE VIBRIO FURNISSII GENOME

We performed in silico analyses of all predicted protein sequences. Gene products were scanned for clusters of orthologous groups (COG) and were mapped to COG categories using AutoFACT software (Tatusov et al., 2000; Koski et al., 2005). The possible functions of each translated polypeptide were determined by comparing the $V$. furnissii proteome to the Pfam-A database (Bateman et al., 2000; Finn et al., 2006) and to the Kyoto 
Encyclopedia of Genes and Genomes (KEGG) database (Kanehisa et al., 2002). Transmembrane helices (TMH) were predicted using Phobius software (Kall et al., 2004, 2007) and the genome was scanned for tRNAs and tmRNAs using tRNAscan-SE 1.23 and ARAGON software respectively. RNAmmer scanned for rRNAs (Lowe and Eddy, 1997; Laslett and Canback, 2004; Lagesen et al., 2007). Results from the in silico proteome analyses were assessed relative to each annotated gene and appropriate comments incorporated into the published genome annotation.

The assembled genome and follow-up analyses results were represented using circos software (Krzywinski et al., 2009).

\section{WHOLE GENOME ALIGNMENTS WITH OTHER VIBRIONACEAE}

The $V$. furnissii genome was aligned against 8 Vibrionaceae genomes representing a selection of human, fish and invertebrate pathogens (i.e., the "emerging Vibrio"), as well as the accepted pathogens $V$. cholerae and $V$. vulnificus, using MUMmer 3.0 software (Delcher et al., 2002). V. furnissii was used as the reference and independently compared to the genomes of $V$. cholerae O1 biovar El Tor strain N16961 (NC002505 and NC002506), V. cholerae O395 (NC009457 and NC009456), V. parahaemolyticus strain RIMD 2210633 (NC004603 and NC004605), V. vulnificus strain CMCP6 (NC004459 and NC004460), V. harveyi strain ATCC BAA-1116 (NC009783, NC009784 and NC009777) and V. splendidus strain LGP32 (FM954972 and FM954973). The genomes of Listonella anguillarum (formerly Vibrio anguillarum) 775 (NC015633 and NC015637) and Photobacterium profundum strain SS9 (NC006370 and NC006371), both members of the Vibrionales were used as outliers. The settings for MUMmer were defined as maxgap $=500$ and mincluster $=100$.

\section{PHYLOGENETIC ANALYSIS}

The phylogeny of each predicted gene product encoded in the $V$. furnissii NCTC 11218 genome was determined using a previously described Perl script that we adapted for use with bacterial genomes (Richards et al., 2009). The script uses the Basic Local Alignment Search Tool (BLAST; $e$-value $1 e^{-20}$ ) to identify local similarities between the amino acid sequence of each CDS and a database containing the protein sequences of 680 bacterial genomes. The amino acid sequences corresponding to the top BLAST hits (range 3-5000) were retrieved from the database and alignments were performed using MUSCLE software. Conserved positions were located with Gblocks software (Talavera and Castresana, 2007) and PhyML software running an approximate likelihood-ratio test (aLRT) algorithm was used to rapidly construct the phylogenetic trees (Guindon and Gascuel, 2003; Anisimova and Gascuel, 2006) using the parameters described in Richards et al. (2009). The phylogenetic trees of all V. furnissii proteins in Newick format are available as supplemental material.

Phylogenetic trees were build using either 16s rRNA nucleotide sequences or nucleotide sequences of the genomic regions of interest R1, R2 and R3, as indicated on Figure 1. This was performed using the programs describe above.

To help establish an even more detailed phylogenetic analysis between $V$. furnissii and the 8 selected Vibrio, the core genome (genes shared by all the strains) and the pangenome (the core genome combined with strain-specific and partially shared genes) were constructed (Tettelin et al., 2008), using a program developed and described by Contreras-Moreira and Vinuesa (2013). In an initial step, the genbank files for each chromosome of each Vibrio species were combined to include the genes of both chromosomes. The second step involved building the core genome and pangenome with default values using the bidirectional best-hit algorithm. In the final step a phylogenetic tree using the provided script was constructed.

\section{DETERMINATION OF GENOMIC ISLANDS}

Genomic islands in the genomes of $V$. furnissii and of the 8 emerging Vibrio were identified using IslandViewer (Waack et al., 2006; Langille et al., 2008; Langille and Brinkman, 2009). Polypeptide sequences within the identified Genomic Islands of $V$. furnissii were compared against all proteins of the 8 , selected Vibrio by BLASTP, and parsed using a $30 \%$ or higher identity filter.

\section{RESULTS}

\section{THE VIBRIO FURNISSII GENOME}

The assembled $V$. furnissii genome is accessible through the NCBI under accession numbers CP002377 (chromosome I) and CP002378 (chromosome II). General features are described in Lux et al. (2011).

The $V$. furnissii genome was aligned against a selection of 8 fully sequenced Vibrio genomes representing the pathogenic Vibrio and 1 outlier. Conserved regions of at least 100 nucleotides in length with at least $80 \%$ nucleotide homology between the $V$. furnissii sequence and each of the compared bacterial species were highlighted, and occur predominantly on chromosome I (Figure 1, track 6-13).

Chromosome I contains 1058 complete CDS with at least $80 \%$ nucleotide homology to other members of the sequenced Vibrionaceae, representing $33.53 \%$ of the total number of CDS located on chromosome I. Conversely, chromosome II contains only 152 complete, homologous CDS, which is $9.87 \%$ (with over $80 \%$ nucleotide homology) of all genes located on chromosome II. Most homology occurred within chromosomes, but in some cases individual CDS appear to have swapped chromosomes; for example, the gene encoding Threonyl-tRNA synthetase (VfuB00041; 44369-46297) is located on chromosome II in the genomes of $V$. furnissii, $V$. cholerae and P. profundum, but is situated on chromosome I of $V$. vulnificus, $V$. parahaemolyticus, $V$. harveyi, V. splendidus and V. fischeri (Figure 1, R7). Conversely, the genes encoding tRNAs for glutamine, lysine and valine are located on chromosome II of $V$. harveyi, but appear on chromosome $\mathrm{I}$ in all the other sequenced Vibrio (Figure 1, track 6-13).

\section{GENOMIC COMPARISONS BETWEEN THE VIBRIONACEAE}

The analyses of nucleotide homology between the sequenced Vibrio enabled us to select 10 regions of particular interest (shaded areas in Figure 1). That are either present in all Vibrio strains or present only in $V$. furnissii.

\section{REGIONS PRESENT IN ALL SELECTED VIBRIO}

Regions R1 (35,611 bp-49,550 bp; VfuA00037-VfuA00045) and R2 (692,714 bp-705,975 bp; VfuA00666-VfuA00691) are highly 


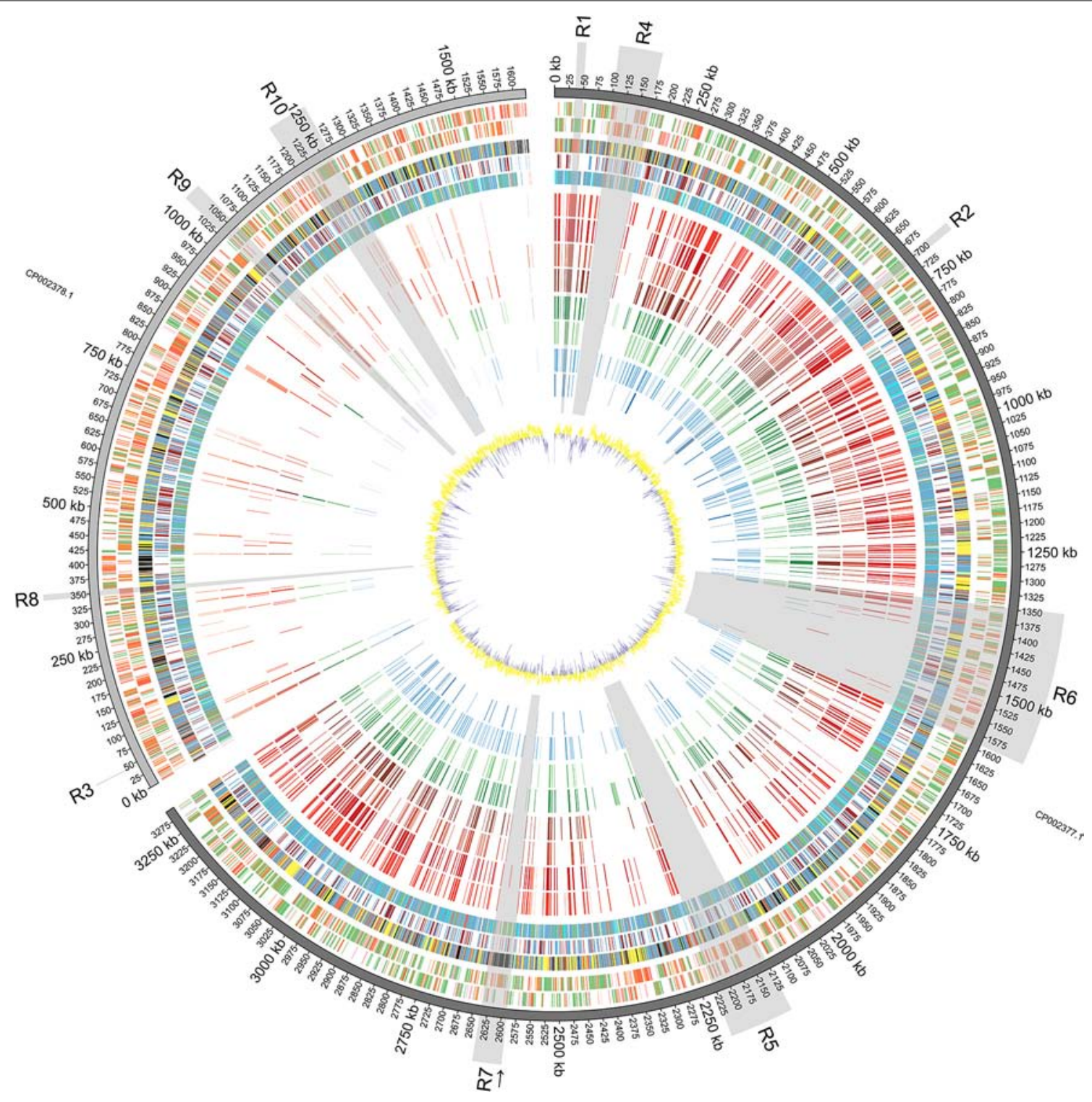

FIGURE 1 | Diagrammatic Representation of the Vibrio furnissii

Genome: From outside to inside: Predicted ORFs on $V$. furnissii forward strand (track 1) and reverse strand (track 2). Green represents 100-80\% amino acid identity; Orange, 80-60\% identity and Red below 60\% identity; COG classification (track 3; Information storage and processing: pink, Cellular processes and signaling: yellow, Metabolism: blue, Poorly characterized: Gray, No hits: black), TMH and SP prediction (track 4; Blue: THM, Light green: SP; Brown: THM+SP), representation of phylogenetic trees (track 5; Red: 3-10 species per leaf; Green: 11-100 species per leaf; Blue: 101-1000 species per leaf; Cyan: 1001 to END species per leaf), NUCMER comparison of 8 bacteria: V. cholerae 01 biovar El Tor strain N16961 (track 6), V. cholerae O395 (track 7), V. parahaemolyticus strain RIMD 2210633 (track 8), V. vulnificus strain CMCP6 (track 9), V. harveyi strain ATCC BAA-1116 (track 10), V. splendidus strain LGP32 (track 11), Listonella (Vibrio) anguillarum 775 (track 12 ) and Photobacterium profundum strain SS9 (track 13), against $V$. furnissii (regions sharing more than $80 \%$ identity are illustrated by bars; red hues represent human associated, green hues represent invertebrate and fish associated, and blue hues represent fish associated pathogens; darker color for identical regions on chromosome I, lighter color for chromosome II), GC plot (track 14; Window size: 10,000, Step size 200). conserved between all the compared sequences. All CDS in either region are present on chromosome I of the compared sequences and fall into the "Information Storage and Processing" COG category. More specifically, region R1 comprises 4 genes that encode tRNAs for threonine, glycine, tyrosine and threonine (VfuAtRNA10-VfuAtRNA13) and genes encoding a DNAdirected RNA polymerase (VfuA00037), 50S ribosomal proteins (VfuA00039-VfuA00042), an elongation factor Tu (VfuA00045), a preprotein translocase (SecE) subunit (VfuA00044) and a transcription anti-terminator (VfuA00043). The encoded peptides have no predicted trans-membrane helices or signal peptide sequences.

Region R2 includes 14 genes that encode 50S ribosomal proteins (VfuA00667-VfuA00670, VfuA00672, VfuA00677VfuA00679, VfuA00682, VfuA00683, VfuA00685, VfuA00686 and VfuA00688) and 8 genes encoding $30 \mathrm{~S}$ ribosomal proteins 
(VfuA00671, VfuA00673, VfuA00676, VfuA00680-VfuA00681, VfuA00684, and VfuA00689-VfuA00690). Genes encoding the preprotein translocase (SecY) subunit and the DNA-directed RNA polymerase subunit alpha (RpoA; VfuA00691) are also located in R2.

Region R3 (44,369 bp-46,297 bp; VfuB00044) also comprises highly conserved genes throughout, and bears a threonyl-tRNA synthetase (VfuB00041), which is located on different chromosomes within the selected Vibrio (see above).

Region R6 (1,347,108 bp to $1,590,549 \mathrm{bp}$; VfuA01296VfuA01532) encompasses genes for flagellar biosynthesis. Several genes encoding the sidophore, vibriobactin, are present.

Region R9 (1,031,067 bp-1,058,755 bp; VfuB00991VfuB01011) has the most homology to other sequenced Vibrio and P. profundum. All CDS in this region are found in the compared Vibrio genomes, although not all CDS contain regions that share at least $100 \mathrm{bp}$ with $80 \%$ homology to the V. furnissii sequence. Those that do, however, include genes encoding 4 subunits of cytochrome O-ubiquinol oxidase (VfuB01000, VfuB01001, VfuB01002, VfuB01003) and protoheme IX farnesyltransferase (VfuB01005).

Region R10 spans from VfuB01141 to VfuB01201 $(1,200,901 \mathrm{bp}-1,265,108 \mathrm{bp})$ and includes 17 hypothetical proteins (VfuB01147, VfuB01172, VfuB01173, VfuB01176, VfuB01177, VfuB01178, VfuB01179, VfuB01180, VfuB01181, VfuB01182, VfuB01183, VfuB01184, VfuB01187, VfuB01188, VfuB01190, VfuB01192 and VfuB01193), two ABC transporters (VfuB01195; VfuB01196; VfuB01197; VfuB01198 and VfuB01154; VfuB01155; VfuB01156; VfuB01158), an operon repressor (VfuB01200), a transcriptional regulator (VfuB01201) for ribose metabolism, 3 transcription factors and regulators (VfuB01145, VfuB01149, VfuB01159, VfuB01165, VfuB01169, VfuB01171, VfuB01186). Bioinformatic analyses show that one of the $\mathrm{ABC}$ transporters is involved in ribose transport, whereas for the second the substrate needs to be identified.

In addition to the CDS in these regions, the V. furnissii genome has 126 other sequences with over $80 \%$ nucleotide homology that are represented in all the sequenced genomes used in this investigation. For example, the gene encoding glyceraldehyde-3phosphate dehydrogenase (VfuA02488) is found in all analyzed strains (Figure 1, highlighted by an arrow).

\section{REGIONS UNIQUE TO VIBRIO FURNISSII}

Regions R4 (101,225 bp- 169,686 bp; VfuA00089-VfuA00153) and R5 (2,125,032 bp-2,226,901 bp; VfuA02027-VfuA02133) are unique to the $V$. furnissii genome, and show no nucleotide homology over $80 \%$ with the other, sequenced Vibrio genomes.

Genes in region R4 encode peptides with highly conserved primary sequence domains and possess peptide homologs throughout the Vibrio. For example, BLAST analysis of the protein sequence of VfuA00116, which encodes UDP-glucose 6-dehydrogenase, revealed the closest related species to be P. profundum SS9. However, the nucleotide composition in R4 is markedly different from the rest of chromosome I, with a G+C content of $43.9 \%$ compared to $50.73 \%$ (Figure 1, track 14).

Region R5 contains a cluster of 13 genes encoding a hydrogenase 4 (VfuA02044-VfuA02062), a putative operon that encodes formate dehydrogenase (VfuA02050-VfuA02054 and VfuA02056) and a sequence encoding a beta-galactosidase (VfuA02089), which are not present in the other Vibrio. The majority of these genes show homology to Aeromonas salmonicida (Reith et al., 2008). This finding is supported by the phylogeny of these proteins (Figure 4) and may have risen through HGT. In addition to these unique sequences, it also contains genes encoding maltose $\mathrm{ABC}$ transporter-associated proteins (VfuA02090-VfuA02094), two tRNAs for Valine (VfuAtRNA63, VfuAtRNA64), a seryl-tRNA synthetase (VfuA02064), a LacIfamily transcriptional regulator (VfuA002095), proteins associated with vitamin B12 synthesis (VfuA02105-VfuA02109) and a galactoside O-acetyltransferase (VfuA02086), that have homologs in other Vibrio but with a nucleotide identity below $80 \%$.

Region R7 (2,591,121 bp-2,637,073 bp; VfuA02488VfuA02545) represents another unique region in the V. furnissii genome. However, in contrast to R4 and R5 is characterized by sequences derived from prophage CTX, and is the only significant phage footprint identified in the $V$. furnissii genome.

One noticeable feature of the $V$. furnissii genome is the presence of one single, large CDS encoding a polypeptide containing 3150 amino acids and with a predicted molecular mass of $329.18 \mathrm{kDa}$ (Region R8 (345,688 bp-355,137 bp; VfuB00340)). The protein is also predicted to contain one signal peptide domain with no trans-membrane helix and 16 VCBS domains (Hemolysin-type calcium-binding repeat) (Baumann et al., 1993). VCBS domains are features of the RTX toxins, a diverse group of pore-forming exotoxins that are synthesized by many gram-negative bacteria and include cytolytic toxins, metalloproteases and lipases (Rodkhum et al., 2006; Frans et al., 2011; Satchell, 2011).

\section{VIBRIO FURNISSII PHYLOGENY}

Given the level of observed homology between the emergent Vibrio, the sequences present in R1 and R2 (Chromosome I), and R3 (Chromosome II) are ideal candidates for confirming phylogenetic relationships that have been established using $16 \mathrm{~S}$ rRNA homology. Although R9 shows a high homology between the selected genomes, this region was not included because the concatenated nucleotide sequence failed to build a phylogenic tree. We therefore constructed a phylogenetic tree using all of the sequence information in regions R1, R2 and R3 (a total of 36 sequences) and constructed a dendogram using the Vibrionaceae $16 \mathrm{~S}$ rDNA sequences ( 9 sequences). Additionally, we constructed a phylogenetic tree based on a pangenome analysis including both chromosome sequences of 9 Vibrio strains. Comparison between the three trees (Figure 2) revealed comparable phylogenetic relationships between P. profundum, V. parahaemolyticus, V. harveyi and V. vulnificus. However, the positions of V. splendidus and Listonella anguillarum vary more in each of the three trees (Figure 2). Most significantly, our analysis suggests a closer grouping between $V$. furnissii and the two $V$. cholerae strains than when $16 \mathrm{~S}$ rRNA sequences, alone, are used.

A systematic analysis of the phylogeny of each $V$. furnissii CDS was performed to determine their possible function and identify those sequences that are uniquely represented in V. furnissii. To illustrate this analysis, the number of bacterial species in the NCBI 

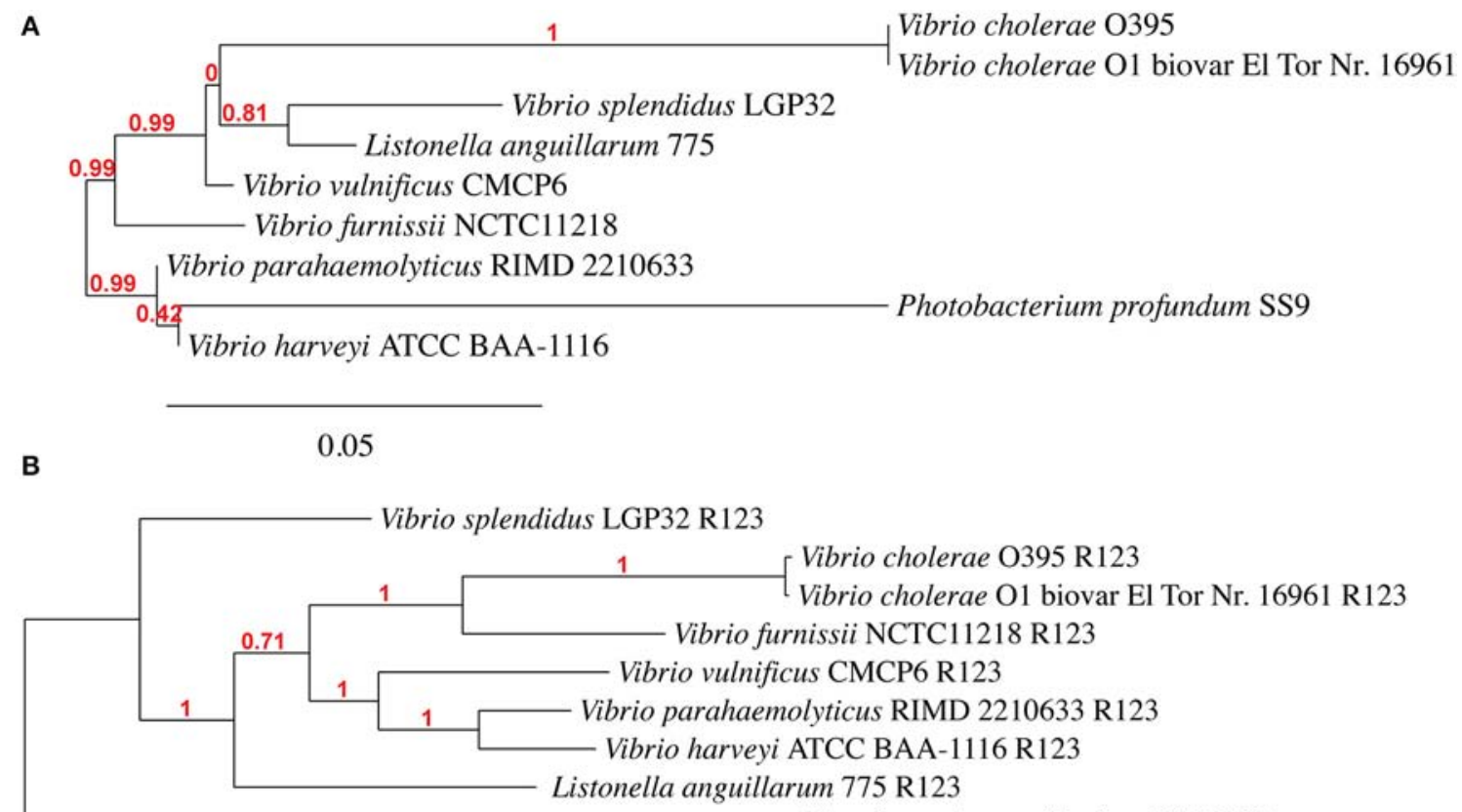

Photobacterium profundum SS9 R123

C

0.1

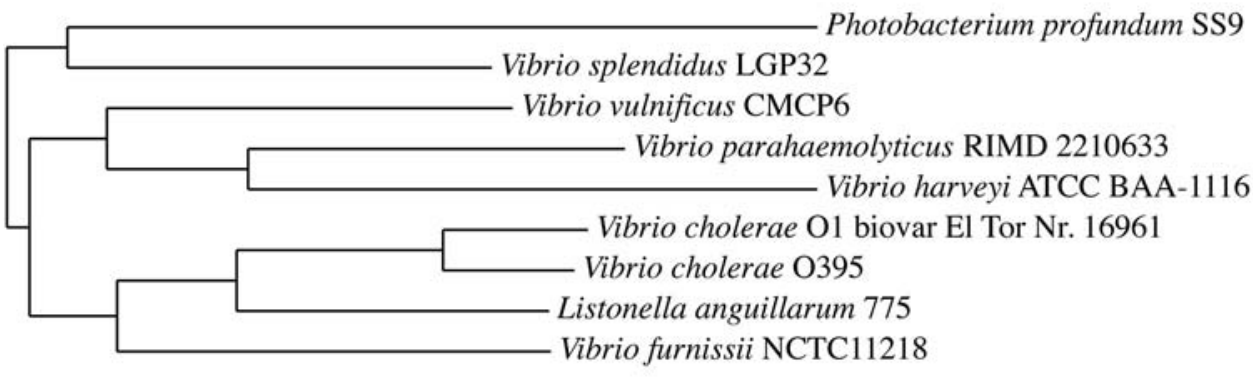

1000.

FIGURE 2 | Phylogenetic trees based on (A) 16S rRNA, (B) from concatenated nucleotide sequences from regions $R 1, R 2$ and $R 3$ and (C) based on Vibrio pangenome from V. furnissii NCTC 11218, V. cholerae 01 biovar El Tor Nr. 16961, V. cholerae 0395, V. parahaemolyticus RIMD 2210633, V. vulnificus CMCP6, V. harveyi ATCC BAA-1116, V. splendidus LGP32, L. anguillarum (formerly $V$. anguillarum) 775 and $P$. profundum SS9. database with sequences homologous to that of the interrogating $V$. furnissii CDS was plotted against the number of CDS with an equal amount of species. For further characterisation the CDS were matched to COG categories.

This distribution of phylogenetic trees can be divided into 4 ranges: 3-10 species per branch, 11-100 species per branch, 1011000 species per branch and more than 1001 to species per branch (Figure 1, track 5: Red: 3-10; Green: 11 to; Blue: 101 to; Cyan: more than 1000).

353 CDS of chromosome I and 195 of chromosome II fall into the lowest category (from 3 to 10). The minimum of 3 was chosen, to ensure the generation of meaningful trees. Most proteins can be categorized as hypothetical and flagellar related. 14.7\% (Chromosome II: $2.6 \%$ ) of the proteins in this group belong to metabolism, $0.8 \%$ (Chromosome II: $0 \%$ ) to cellular processes and signaling and 5.9\% (Chromosome II: 23.6\%) are grouped to information storage and processing. $78.6 \%$ (Chromosome II: $73.8 \%$ ) have not been assigned a COG category.

The second, or lower mid-range (from 11 to 100) category contains 638 CDS of chromosome I and 412 of chromosome II. These are mainly hypothetical proteins. On Chromosome I $30.0 \%$ (Chromosome II: $15.5 \%$ ) of the proteins within this range are in the metabolism COG set, 3.0\% (Chromosome II: $0.7 \%$ ) are cellular processes and signaling and $12.5 \%$ (Chromosome II: $28.2 \%$ ) are for information storage and processing. Again, the majority (Chromosome I: 54.5\%/Chromosome II: 55.6\%) are not categorized.

The range from 101 to 1000 contains 1491 CDS from chromosome I and 511 CDS from chromosome II. Amongst these are ribosomal sequences that are classified as several tRNA 
synthetases. COG set metabolism makes up to $68.6 \%(25.2 \%)$, $2.3 \%(1.1 \%)$ are cellular processes and signaling related and 9.3\% $(41.0 \%)$ are information storage and processing. Only $19.8 \%$ have not assigned a COG term, contrasting with $32.7 \%$ on chromosome II.

The last category is composed mainly of proteins within the COG sets metabolism $(80 \% / 17.7 \%)$, cellular processes, and signaling $0.8 \%(0 \%)$ and metabolism $10.4 \%$ (55.8\%), such as ABC-Transporters or proteins involved in fatty acid and cell wall biosynthesis. Not categorized are only $8.8 \%(26.5 \%)$ of the proteins.

\section{THE SUPER INTEGRON OF THE V. FURNISSII}

The Super Integron (SI) is a common feature amongst the Vibrio species, but it is also highly variable. The Super Integron of Vibrio furnissii is situated on chromosome II and encompasses the CDS VfuB01511 and VfuB00281 $(1,591,485-281,565)$ stretching over a total of $311,971 \mathrm{bp}$. This is largest SI identified in any Vibrio strain. The V. furnissii SI starts with site-specific recombinase IntIA (VfuB01511) and terminates at VfuB00281, an anaerobic ribonucleoside-triphosphate reductase. The SI also encompasses $3 \mathrm{attC}$ sites, which are a characteristic feature of Super Integrons. A third of the CDS within the SI are annotated as hypothetical, but many could be ascribed a function. These are mainly ABC transporters. A more detailed comparison between the $V$. furnissii and the $V$. cholerae SIs shows a high degree of conservation between both elements (Figure 3 ). However, it is clearly visible by the disruption at the end of the SI that a high amount of genomic rearrangements occurred in this region.

\section{PATHOGENIC AND VIRULENCE RELATED FEATURES OF THE $\boldsymbol{v}$. FURNISSII GENOME}

Based on established studies, we scanned the $V$. furnissii sequence for virulence-related features including quorum sensing, biofilm formation, virulence factors, chitin metabolism and natural competence.

Many bacterial species communicate by the secretion and detection of small signaling molecules called autoinducers. In $V$. furnissii two different types of quorum sensing mechanisms are present. The CAI-1 autoinducer, a substituted 13-carbon alkane, is produced by VfuB00269 (CqsA) and detected by VfuB00270 (CqsS), both located on chromosome II. The second autoinducer AI-2, a furanosyl borate, is produced by VfuA02965 (LuxS) on chromosome I and detected by VfuB00359, VfuB00358 (LuxP, LuxQ) on chromosome II.

In addition LuxU (VfuA02581) is phosphorylated by the unbound sensor kinases CqsS and LuxP/Q, which in turn phosphrylates LuxO (VfuA2580). The genes encoding LuxU/O are located on chromosome I in $V$. furnissii.

The lux-Operon is reported to be closely linked with HapR (VfuA00883), which controls biofilm formation by repressing the $v p s \mathrm{~T}$ transcription factor (VfuB00730) and controlling expression of genes for products that alter intracellular c-diGMP second messenger molecules (Tsou et al., 2009). Based on the $V$. cholerae QS model, biofilm formation requires Qrr-dependent

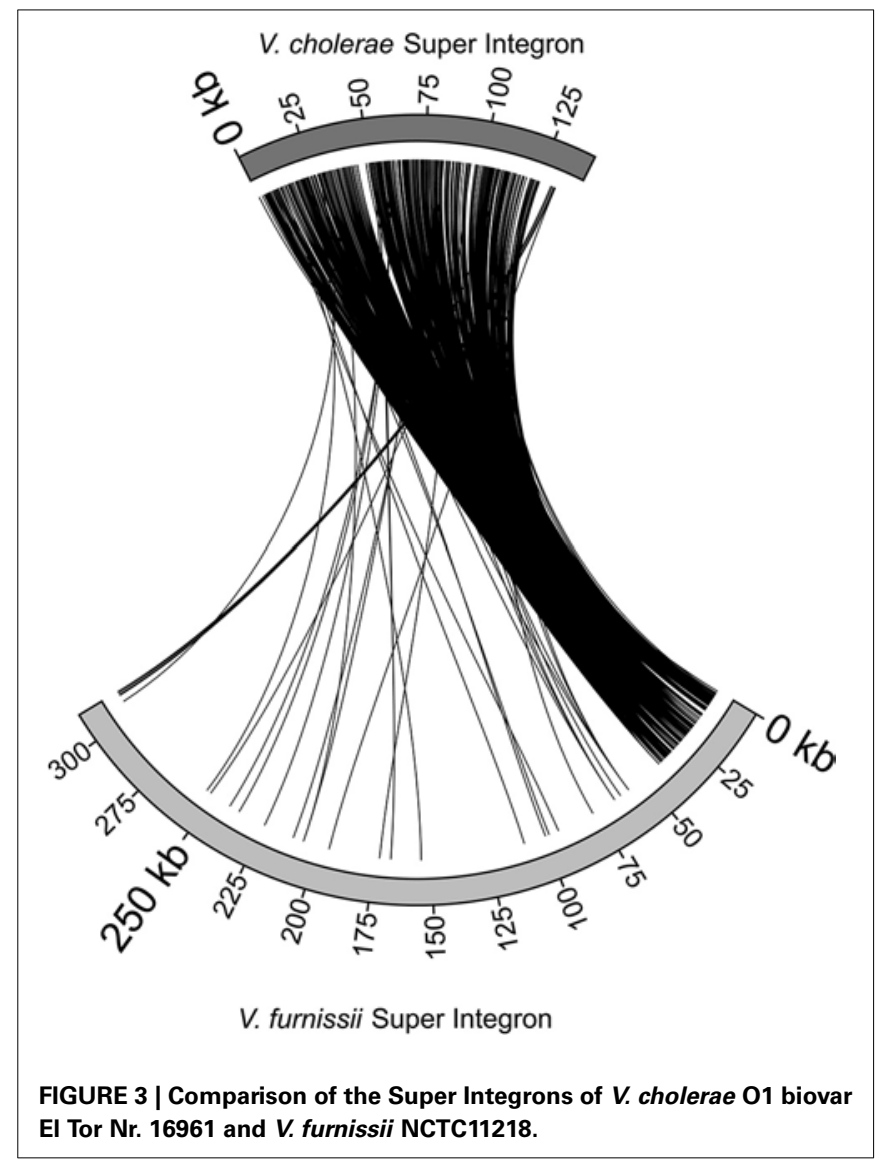

repression of hapR translation to permit expression of the $v p s \mathrm{~L}-\mathrm{N}$ exopolysacharide biosynthesis genes (Bardill et al., 2011). HapR is a TetR family transcriptional regulator that has been shown to regulate a variety of phenotypes important for both virulence and environmental survival (Tsou et al., 2011). VpsT is located on chromosome II of $V$. furnissii.

The major pilin subunit tcpA (VfuA00872) is present in V. furnissii. Being part of the toxin co-regulated pilus (TCP), it has been reported as a critical colonization factor (Manning, 1997).

$V$. furnissii has been reported to be a chitinovorous marine bacterium (Yu et al., 1987), (Bassler et al., 1991a,b; Yu et al., 1991) and there is bioinformatic evidence of at least one extracellular chitinase ChiA-2 (VfuB01027). Furthermore, a chitinase is encoded by $v f u A 02755$ and $v f u B 00330$ encodes for a chitodextrinase. The $\mathrm{N}$-acetylglucosamine-binding protein $\mathrm{A}$ is also present in V. furnissii (VfuB01209).

\section{GENOMIC AND PATHOGENICITY ISLANDS OF THE V. FURNISSII GENOME}

The presence of genomic islands in the sequenced Vibrio was determined using IslandPick, SIGI-HMM and IslandPathDIMOB (summarized in Table $\mathbf{1}$ ).

In all Vibrio species analyzed, GEIs could be identified by the SIGI-HMM and/or the Islandpath-DIMOB methods. IslandPick could only identify GEIs in V. parahaemolyticus, V. vulnificus and $V$. harveyi. The highest number of GEIs was identified using 
Table 1 | Number of identified GEI in V. furnissii NCTC 11218, V. cholerae 01 biovar EI Tor Nr. 16961, V. cholerae 0395, V. parahaemolyticus RIMD 2210633, V. vulnificus CMCP6, $V$. harveyi ATCC BAA-1116, V. splendidus LGP32, L. anguillarum 775 and P. profundum SS9.

\begin{tabular}{|c|c|c|c|c|c|c|}
\hline \multirow[t]{3}{*}{ Chromosome } & \multicolumn{6}{|c|}{ Identification method } \\
\hline & \multicolumn{2}{|c|}{ SIGI-HMM } & \multicolumn{2}{|c|}{ Islandpath-DIMOB } & \multicolumn{2}{|c|}{ IslandPick } \\
\hline & $\mathbf{I}$ & II & $\mathbf{I}$ & II & $\mathbf{I}$ & II \\
\hline V. furnissii & 6 & 5 & 4 & - & - & - \\
\hline V. cholerae & 11 & 5 & 2 & 3 & - & - \\
\hline V. cholerae 0395 & 9 & 6 & 3 & 4 & - & - \\
\hline V. parahaemolyticus & 16 & 6 & 5 & 3 & 5 & 12 \\
\hline V. vulnificus & 17 & 5 & 4 & 3 & 4 & - \\
\hline V. splendidus & 24 & 10 & 4 & 2 & - & - \\
\hline V. harveyi & 30 & 2 & 15 & 10 & 9 & - \\
\hline L. anguillarum & 19 & 8 & 7 & 2 & - & - \\
\hline P. profundum & 12 & 17 & 7 & 4 & - & - \\
\hline
\end{tabular}

SIGI-HMM software. The highest number of proteins within identified GEIs is in V. harveyi chromosome I (434), the lowest in $V$. furnissii and $V$. cholerae (both 114). On chromosome II the highest number of proteins is in V. parahaemolyticus (355), the lowest in V. furnissii (19). Most proteins within these GEIs are annotated as hypothetical proteins, transposases or ribosomal proteins and are present in all Vibrio. Interestingly, region R5 and R6 cover many CDS that are part of the predicted GEIs. The phylogenetic trees of these proteins show the variability and conservation of these regions between the different Vibrios. For instance VfuA01497, a transcriptional regulator belonging to the TetR family, (Figure 5F) is only present in the two $V$. cholerae strains, whereas VfuA01991, a E1-E2 family cation transport ATPase, seems to be closely related to $V$. harveyi and $V$. parahaemolyticus (Figure 5E). Similar evidence for HGT can be found throughout all predicted GEIs.

\section{DISCUSSION}

Our analysis of the distribution of open reading frames of the $V$. furnissii genome emphasizes two aspects of Vibrio genetics. First, the majority of the predicted coding sequences with over $80 \%$ similarity to other genes is located on chromosome I. Second, the proportion of CDS that display $60 \%$ or less identity to previously identified genes are predominantly found on chromosome II. This distribution is consistent with previous observations that the larger of the two chromosomes in Vibrio predominantly contains genes encoding functions corresponding to growth and survival under standard conditions, such as housekeeping genes, which are less likely to be divergent (Dryselius et al., 2007). In other Vibrio species, genes that encode functions beneficial for unusual growth situations tend to be located on the smaller chromosome (Jain et al., 2002; Philippe and Douady, 2003; Dryselius et al., 2007). Also there is a high representation of COGs in the "metabolism" set compared to other Vibrio which may indicate that $V$. furnissii has a greater than expected capacity to adapt to, and exploit, diverse environmental niches. Additionally the CDS present on chromosome II tend to be shorter and more often disrupted by random nucleotide changes than the CDS present on chromosome I, which is compounded by a highly variable $\mathrm{G}+\mathrm{C}$ content on chromosome II (Figure 1, track 1/2-red group; track 14). Possible explanations for this difference in gene organization between the V. furnissii chromosomes include a lesser selective pressure on chromosome II and/or a lesser need for repair which, taken together, would result in an increased possibility of retained mutations on chromosome II, generating novel genes (Garcia-Vallve et al., 2000; Dryselius et al., 2007). It is also possible that, under certain conditions, differences in the copy number of predominantly "housekeeping" chromosome I and "variable" chromosome II might occur, potentially increasing the effective level of expression of genes on chromosome II, to the bacterium's advantage (Heidelberg et al., 2000; Schoolnik and Yildiz, 2000; Jain et al., 2002; Philippe and Douady, 2003; Dryselius et al., 2007). Also, the G+C content of the Vibrio furnissii genome is $4-10 \%$ higher than other sequenced Vibrio species, possibly indicating higher levels of HGT (GarciaVallve et al., 2000) in V. furnissii than in the other, sequenced Vibrio. This is also supported by the position of the predicted GEIs, which fall in regions with a change in $\mathrm{G}+\mathrm{C}$ contents (Figure 1, track 14).

It is well established that proteins or enzymes that interact directly with DNA are under high selection pressure, and therefore it is not surprising that the homology between these genes is so high across the sequenced genomes. This level of conservation exists across the entire bacterial phylogeny, as evidenced by the fact that all genes in R1 and R2 have 101-1000 sequences in their respective phylogenetic trees (Figure 1, track 5- blue group) except for VfuA00671 which encodes the 30S ribosomal protein S19, and has only 11-100 homologous sequences in the phylogeny (Figure 1, track 5-blue group).

It is plausible that the divergence of $\mathrm{R} 4$ arose through the accumulation of silent mutations in an unusually A+T rich portion of the $V$. furnissii genome. Novel features of $V$. furnissii are clearly the presence of a hydrogenase 4 and formate dehydrogenase (see Region R5), which have high homology to Aeromonas salmonicida (Figure 4), although their functions in V. furnissii remain unclear. Consequently, R5 contains suites of genes that are unique to $V$. furnissii and probably arose via HGT, interspersed with sequences that have homologs (albeit poor homologs) within the other Vibrio. Interestingly genomic islands have been predicted in region 5 . These are fundamental differences between the sequences in R4 and R5 that are suggestive of different evolutionary histories between these regions.

Our analyses of the phylogeny of the selected 9 Vibrio strains reveals that the position of V. splendidus and Listonella anguillarum within a phylogenetic tree is dependent on the method used to construct the phylogenetic tree. This observation can possibly be explained by variations within the smaller chromosome, although more work must be done to support this hypothesis. A second observation is that $V$. furnissii groups more firmly within the $V$. cholerae group than a phylogenic analysis based only on $16 \mathrm{~S}$ rRNA sequences suggests. 

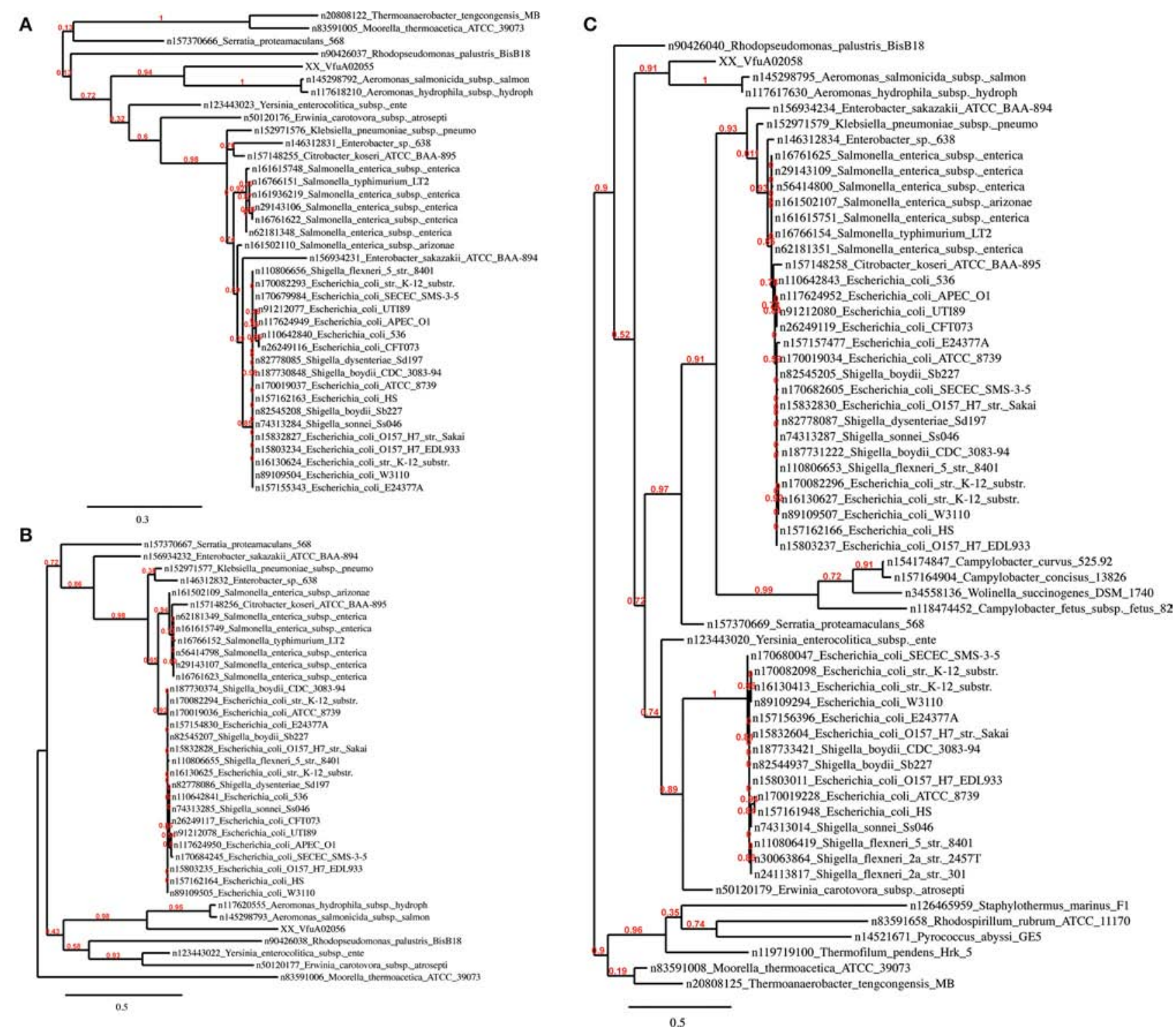

FIGURE 4 | Phylogenetic trees of the V. furnissii proteins (A) VfuA02055, (B) VfuA02056, and (C) VfuA02058 showing their homology to Aeromonas salmonicida. Numbers representing branch support values.

The super integron common in Vibrio is also present in $V$. furnissii. Although the $V$. furnissii super integron still shows characteristic features, such as the attC sites, it has an increased size and many genomic rearrangements. This underlines the high genomic variability of the SI region.

Amongst the novel features shown by our analysis is VfuB00340 located on chromosome II, which is likely to belong to the RTX protein family. Homologs to putative RTX toxins have been identified in $V$. vulnificus and may play a role in cell adhesion and/or pathogenesis. In $V$. anguillarum, RTX toxins are a major component of virulence. A phylogenetic analysis of the amino acid sequence of VfuB00340 failed to return any homologous sequences. However, the presence of calcium binding sites and von Willebrand factor type A, and repetitive VCBS domains indicates that VfuB00340 may be a novel toxin of the RTX protein family, although further in vivo analysis is required to confirm its precise function. One of the supposed functions of the RTX protein is be to support haemolysis. The major virulence factor of Vibrios, haemolysin, encoded by the gene hylA is present in V. furnissii (VfuB01125) and located on chromosome II (Li et al., 2008) demonstrated contribution of RTX toxins to haemolytic activity in L. anguillarum. Although a $r t x$ gene cluster is not present as such, $V$. furnissii possesses upstream of VfuB0340 several genes that could be involved in this novel toxic activity (VfuB0341VfuB0347), such as two RTX toxin transproters (VfuB00343 and VfuB00346). However, the sequence encoding the toxin secretion transporter is missing from the $V$. furnissii genome, although it is quite possible that its function is performed by another secretion protein. 
There have been reports linking Vibrio motility to virulence, with motility being down-regulated and virulence factor expression simultaneously up-regulated. Silva et al., observed that transcription of tox $\mathrm{T}, \operatorname{ct} x \mathrm{~A}$, and tcpA is up-regulated in a V. cholerae non-motile $(\operatorname{mot} \mathrm{Y})$ strain (Silva et al., 2006). Supporting evidence for this model comes from Ghosh et al., who found that the histone-like nucleoid structuring protein H-NS stimulates motility by stimulating flrA expression while repressing $c t x \mathrm{AB}$ and $t c p \mathrm{~A}$ transcription (Ghosh et al., 2006).

In response to the appropriate environmental signal(s), tcpA is stimulated by TcpI, while simultaneously reducing Vibrio chemotaxis-directed motility (Harkey et al., 1994). In contrast to $V$. cholerae, tcpI is located on the second chromosome of V. furnissii (VfuB00809). TcpI was identified as a ToxR-activated gene that encodes an inner membrane protein with extensive sequence similarity to the highly conserved signaling domain in methyl-accepting membrane chemoreceptors and plays an important role in colonization of the small bowel. Both $V$. cholerae regulatory proteins ToxR and ToxT, which are involved in activation of various virulence genes, such as the above-mentioned CT (Miller et al., 1987; Krukonis et al., 2000) or the expression of OmpU (VfuA00928) that mediates resistance to bile and anionic detergents (Mathur and Waldor, 2004), are also absent in $V$. furnissii. However, the regulatory protein ToxS, a protein known to interact with ToxR and stimulate ToxR activity, is present in V. furnissii (VfuA02617) (DiRita and Mekalanos, 1991). This observation suggests the presence of an unknown protein carrying out a function similar to VcToxR, in V. furnissii.

Bioluminescence, which has been well characterized in $V$. harveyi, is missing in V. furnissii. However, some genes belonging to the lux family and related quorum sensing systems are present. These genes are closely linked to the Vibrio competence system. This competence system is also dependent on chitin utilization. Chitin, composed of 1,4-linked GlcNAc residues, induces the expression of a 41-gene regulon in $V$. furnissii that is involved in chitin colonization, digestion, transport, and assimilation, and includes genes predicted to encode a type IV pilus assembly complex (Meibom et al., 2004, 2005). The presence of several chitinase and associated genes in $V$. furnissii confirms previous reports and suggests $V$. furnissii to be naturally competent. However, two additional chitinases found in V. cholerae are lacking in V. furnissii. In the presence of chitin, TfoX (VfuA01621) is induced (Yamamoto et al., 2011) and is thought to play a role in controlling the transcription of comEA (Bardill et al., 2011). The com-system is essential for natural competence (i.e., cells' ability to uptake DNA from the environment) and incorporation of extracellular DNA into the genome which is one of the driving motors for HGT and the generation of genomic islands (Scrudato and Blokesch, 2012).

Bacterial genomes typically harbor a variable number of accessory genes acquired by HGT that encode adaptive traits that are beneficial to the host under certain growth or environmental conditions (Schmidt and Hensel, 2004). These so called Genomic Islands (GEIs) are clusters of genes that are typically recognized as discrete DNA segments between closely related bacterial strains. It is widely recognized that HGT has played a crucial role in the evolution of bacterial species. Furthermore, several lines of evidence suggest the existence of evolutionary ancient GEIs spread over versatile groups of otherwise unrelated bacteria (Juhas et al., 2009). Evidence therefore suggests that the formation of GEIs contributes to the diversification and adaptation of microorganisms, and the presence of GEIs may confer a significant impact on genome plasticity and evolution, the dissemination of antibiotic resistance and virulence genes, and the formation of catabolic pathways (Juhas et al., 2009). The sequences of GEIs display certain properties that mark them as being atypical compared to the overall genome of the organism in which they are found. These features include a large chromosomal region present in a subset of isolates of a given species and absent from other isolates of the same species; the presence of loci involved in genomic mobility such as integrases and transposases; the association with one or more tRNA genes; the presence of flanking direct-repeat sequences that mark the site where incoming DNA recombined with the host genome; a $\mathrm{G}+\mathrm{C}$ content that differs significantly from that of the host organism; and an instability in chromosomal insertion sites. The formation of GEIs is still poorly understood, although at least one of three primary mechanisms, conjugation, transduction, and transformation, is believed to be involved (Juhas et al., 2009). GEIs are classified based on the different functions they encode, which include metabolic islands, degradation islands, resistance islands, symbiosis islands, and pathogenicity islands (Murphy and Boyd, 2008).

As the nomenclature suggests, pathogenicity islands (PAIs) are unstable chromosomal regions that bear virulence-related genes and are therefore associated with different virulence-associated characteristics and phenotypes. The PAIs that arose through HGT generally exhibit a $\mathrm{G}+\mathrm{C}$ content that diverges from that of the host genome, a gene or genes encoding a P4-like integrase, and a chromosomal insertion at a tRNA-serine locus that is flanked by direct repeats (Groisman and Ochman, 1996; Dobrindt et al., 2004). Out of the 114 proteins within the identified GEIs on $V$. furnissii genome, only 63 (see supplement) could be allocated with phylogenetic trees. The proteins with no tree are mainly hypothetical proteins, which typically have no or a low branched tree. One other possibility is that there are too many species involved in tree building, which will also cause the pipeline program to fail. For instance, one hypothetical protein (VfuA00164) has a higher homology to V. harveyi, V. splendidus, L. anguillarum, and $P$. profundum than to $V$. cholerae. These 4 species are marine bacteria and the protein may therefore have a function that is pertinent to the marine pathogens. One other particularly interesting protein is VfuB00210 (Figure 5D), only present in V. furnissii, also a hypothetical protein with a conserved domain of unknown function (COG4694). Further determination is therefore required to ascribe functional reference to theses identified sequences.

Evidence for horizontal gene transfer not only comes from the identification of GEIs in the $V$. furnissii genome, but can be also found in the phylogenic trees. The protein VfuA00259 (a cell wall-associated hydrolase) is deduced to be related to Hahella chejuensis, a marine proteobacterium (Figure 5C). As already stated above, Aeromonas salmonicida may have been the donor for various transfer events (Figure 4). The phylogenetic trees also suggest gene transfer between more closely related strains such as $P$. profundum and $V$. furnissii 

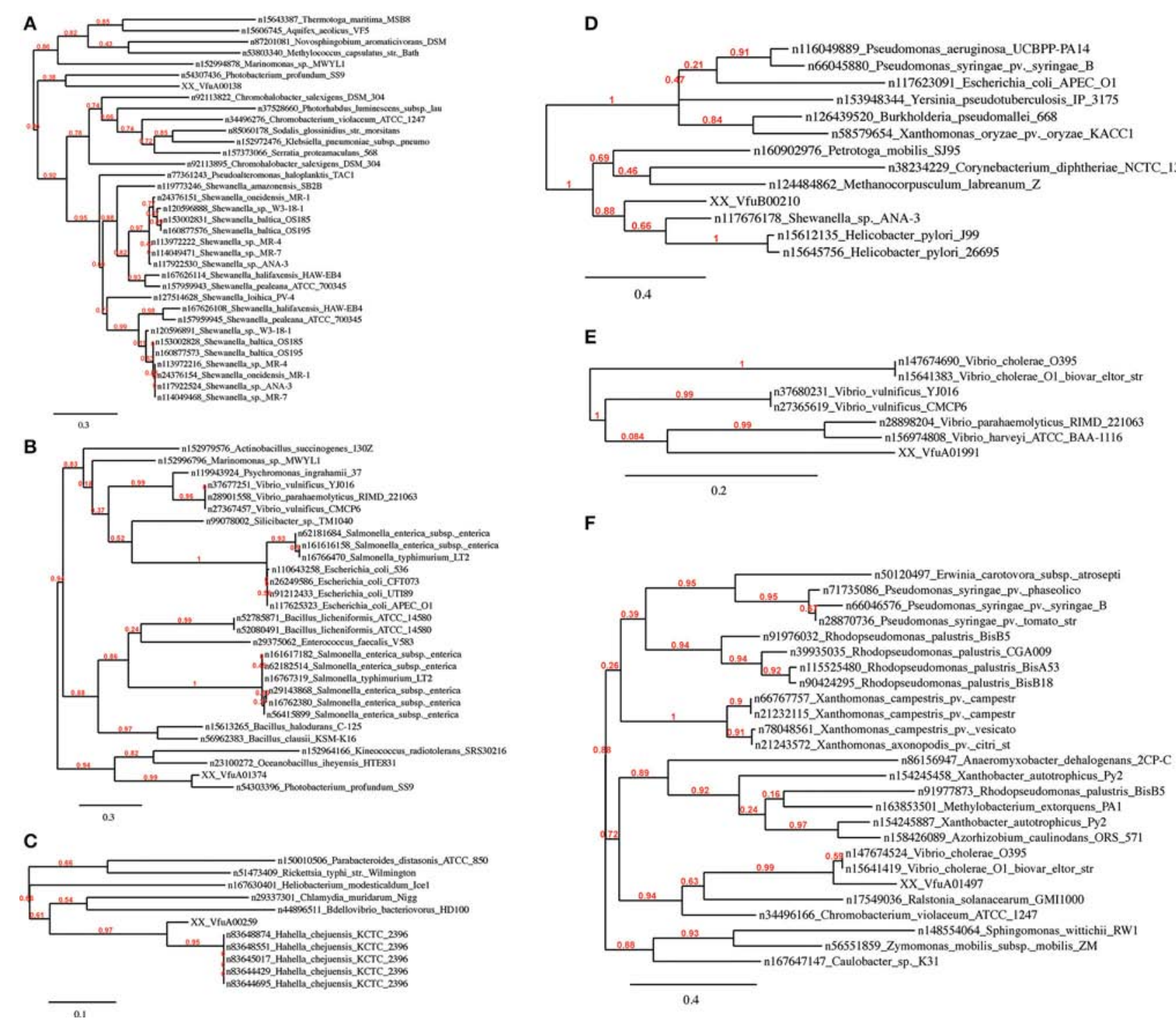

E

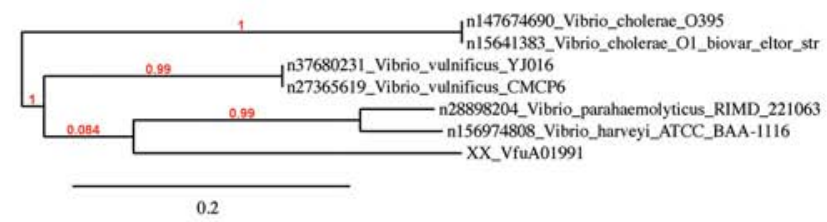

$\mathbf{F}$

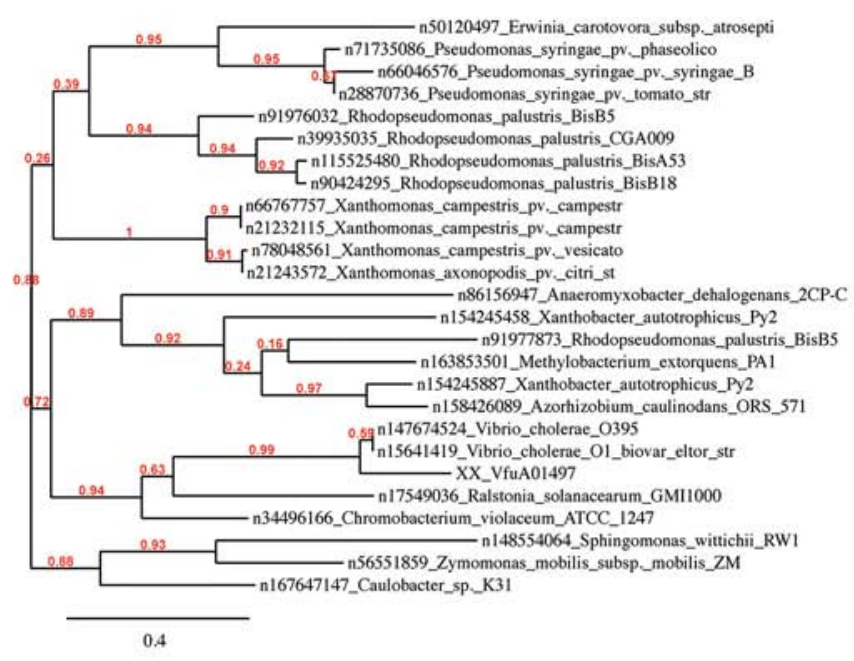

FIGURE 5 | Phylogenetic trees of the V. furnissii proteins (A) VfuA00138, (B) VfuA01374, (C) VfuA00259, (D) VfuB00210, (E) VfuA01991, and (F) VfuA01497 showing putative horizontal gene transfer events. Numbers representing branch support values.

(Figures 5A,B). These results suggest a high frequency of HGT between $V$. furnissii and bacteria that occupy a similar ecological niche.

Even if none of the major pathogenicity islands are present in $V$. furnissii, it cannot be ruled out that $V$. furnissii bears a pathogenic potential for humans and, more immediately, to marine arthropods which are the basis of marine economic activity in regions that are already vulnerable to climate change. The presence of a novel RTX type protein is clear evidence of this potential. Furthermore, the appearance of hydrogenase 4 and formate dehydrogenase, which originated from non-closely related bacteria, alongside functional QS and competence systems shows a high potential to assimilate virulence related genes through HGT and therefore quickly increase the pathogenicity of V. furnissii.

\section{ACKNOWLEDGMENTS}

We are extremely grateful to Darren M. Soanes (University of Exeter) for providing the phylogeny Perl script. This research was funded by a grant from Shell Research Ltd.

\section{SUPPLEMENTARY MATERIAL}

The Supplementary Material for this article can be found online at: http://www.frontiersin.org/journal/10.3389/fmicb. 2014.00435/abstract

\section{REFERENCES}

Anisimova, M., and Gascuel, O. (2006). Approximate likelihood-ratio test for branches: a fast, accurate, and powerful alternative. Syst. Biol. 55, 539-552. doi: $10.1080 / 10635150600755453$

Austin, B. (2010). Vibrios as causal agents of zoonoses. Vet. Microbiol. 140, 310-317. doi: 10.1016/j.vetmic.2009.03.015 
Bardill, J. P., Zhao, X., and Hammer, B. K. (2011). The Vibrio cholerae quorum sensing response is mediated by Hfq-dependent sRNA/mRNA base-pairing interactions. Mol. Microbiol. 80, 1381-1394. doi: 10.1111/j.1365-2958.2011. 07655.x

Bassler, B., Gibbons, P., Yu, C., and Roseman, S. (1991a). Chitin utilization by marine bacteria. Chemotaxis to chitin oligosaccharides by Vibrio furnissii. J. Biol. Chem. 266, 24268-24275.

Bassler, B., Yu, C., Lee, Y., and Roseman, S. (1991b). Chitin utilization by marine bacteria. Degradation and catabolism of chitin oligosaccharides by Vibrio furnissii. J. Biol. Chem. 266, 24276-24286.

Bateman, A., Birney, E., Durbin, R., Eddy, S., Howe, K., and Sonnhammer, E. (2000). The Pfam protein families database. Nucleic Acids Res. 28, 263-266. doi: 10.1093/nar/28.1.263

Baumann, P., Baumann, L., Woolkalis, M. J., and Bang, S. S. (1983). Evolutionary relationships in vibrio and Photobacterium: a basis for a natural classification. Annu. Rev. Microbiol. 37, 369-398. doi: 10.1146/annurev.mi.37.100183. 002101

Baumann, U., Wu, S., Flaherty, K. M., and McKay, D. B. (1993). Threedimensional structure of the alkaline protease of Pseudomonas aeruginosa: a two-domain protein with a calcium binding parallel beta roll motif. EMBO J. 12, 3357-3364.

Ben-Haim, Y., Thompson, F. L., Thompson, C. C., Cnockaert, M. C., Hoste, B., Swings, J., et al. (2003). Vibrio coralliilyticus sp. nov., a temperature-dependent pathogen of the coral Pocillopora damicornis. Int. J. Syst. Evol. Microbiol. 53, 309-315. doi: 10.1099/ijs.0.02402-0

Chakraborty, S., Nair, G. B., and Shinoda, S. (1997). Pathogenic vibrios in the natural aquatic environment. Rev. Environ. Health 12, 63-80. doi: 10.1515/REVEH.1997.12.2.63

Colwell, R. R. (2004). Infectious disease and environment: cholera as a paradigm for waterborne disease. Int. Microbiol. 7, 285-289.

Contreras-Moreira, B., and Vinuesa, P. (2013). GET_HOMOLOGUES, a versatile software package for scalable and robust microbial pangenome analysis. Appl. Environ. Microbiol. 79, 7696-7701. doi: 10.1128/AEM.02411-13

Dalsgaard, A., Glerup, P., Hoybye, L., Paarup, A., Meza, R., Bernal, M., et al. (1997). Vibrio furnissii isolated from humans in Peru: a possible human pathogen? Epidemiol. Infect. 119, 143-149. doi: 10.1017/S095026889700798X

Delcher, A., Phillippy, A., Carlton, J., and Salzberg, S. (2002). Fast algorithms for large-scale genome alignment and comparison. Nucleic Acids Res. 30, 2478-2483. doi: 10.1093/nar/30.11.2478

DiRita, V. J., and Mekalanos, J. J. (1991). Periplasmic interaction between two membrane regulatory proteins, ToxR and ToxS, results in signal transduction and transcriptional activation. Cell 64, 29-37. doi: 10.1016/0092-8674(91)90206-E

Dobrindt, U., Hochhut, B., Hentschel, U., and Hacker, J. (2004). Genomic islands in pathogenic and environmental microorganisms. Nat. Rev. Microbiol. 2, 414-424. doi: 10.1038/nrmicro884

Dryselius, R., Kurokawa, K., and Iida, T. (2007). Vibrionaceae, a versatile bacterial family with evolutionarily conserved variability. Res. Microbiol. 158, 479-486. doi: 10.1016/j.resmic.2007.04.007

Finn, R., Mistry, J., Schuster-Bockler, B., Griffiths-Jones, S., Hollich, V., Lassmann, T., et al. (2006). Pfam: clans, web tools and services. Nucleic Acids Res. 34, D247-D251. doi: 10.1093/nar/gkj149

Frans, I., Michiels, C. W., Bossier, P., Willems, K. A., Lievens, B., and Rediers, H. (2011). Vibrio anguillarum as a fish pathogen: virulence factors, diagnosis and prevention. J. Fish Dis. 34, 643-661. doi: 10.1111/j.1365-2761.2011.01279.x

Garcia-Vallve, S., Romeu, A., and Palau, J. (2000). Horizontal gene transfer in bacterial and archaeal complete genomes. Genome Res. 10, 1719-1725. doi: $10.1101 /$ gr. 130000

Gay, M., Renault, T., Pons, A.-M., and Le Roux, F. (2004). Two vibrio splendidus related strains collaborate to kill Crassostrea gigas: taxonomy and host alterations. Dis. Aquat. Org. 62, 65-74. doi: 10.3354/dao062065

Ghosh, A., Paul, K., and Chowdhury, R. (2006). Role of the histone-like nucleoid structuring protein in colonization, motility, and bile-dependent repression of virulence gene expression in Vibrio cholerae. Infect. Immun. 74, 3060-3064. doi: 10.1128/IAI.74.5.3060-3064.2006

Groisman, E. A., and Ochman, H. (1996). Pathogenicity islands: bacterial evolution in quantum leaps. Cell 87, 791-794. doi: 10.1016/S0092-8674(00)81985-6

Guindon, S., and Gascuel, O. (2003). A simple, fast, and accurate algorithm to estimate large phylogenies by maximum likelihood. Syst. Biol. 52, 696-704. doi: $10.1080 / 10635150390235520$
Harkey, C. W., Everiss, K. D., and Peterson, K. M. (1994). The Vibrio cholerae toxincoregulated-pilus gene tcpI encodes a homolog of methyl-accepting chemotaxis proteins. Infect. Immun. 62, 2669-2678.

Heidelberg, J., Eisen, J., Nelson, W., Clayton, R., Gwinn, M., Dodson, R., et al. (2000). DNA sequence of both chromosomes of the cholera pathogen Vibrio cholerae. Nature 406, 477-483. doi: 10.1038/35020000

Hickman-Brenner, F., Brenner, D., Steigerwalt, A., Schreiber, M., Holmberg, S., Baldy, L., et al. (1984). Vibrio fluvialis and Vibrio furnissii isolated from a stool sample of one patient. J. Clin. Microbiol. 20, 125-127.

Jain, R., Rivera, M., Moore, J., and Lake, J. (2002). Horizontal gene transfer in microbial genome evolution. Theor. Popul. Biol. 61, 489-495. doi: 10.1006/tpbi.2002.1596

Juhas, M., van der Meer, J. R., Gaillard, M., Harding, R. M., Hood, D. W., and Crook, D. W. (2009). Genomic islands: tools of bacterial horizontal gene transfer and evolution. FEMS Microbiol. Rev. 33, 376-393. doi: 10.1111/j.15746976.2008.00136.x

Kall, L., Krogh, A., and Sonnhammer, E. (2004). A combined transmembrane topology and signal peptide prediction method. J. Mol. Biol. 338, 1027-1036. doi: 10.1016/j.jmb.2004.03.016

Kall, L., Krogh, A., and Sonnhammer, E. (2007). Advantages of combined transmembrane topology and signal peptide prediction-the Phobius web server. Nucleic Acids Res. 35, W429-W432. doi: 10.1093/nar/gkm256

Kanehisa, M., Goto, S., Kawashima, S., and Nakaya, A. (2002). The KEGG databases at GenomeNet. Nucleic Acids Res. 30, 42-46. doi: 10.1093/nar/30.1.42

Koski, L., Gray, M., Lang, B., and Burger, G. (2005). AutoFACT: an automatic functional annotation and classification tool. BMC Bioinformatics 6:151. doi: 10.1186/1471-2105-6-151

Krukonis, E., Yu, R., and DiRita, V. (2000). The Vibrio cholerae ToxR/TcpP/ToxT virulence cascade: distinct roles for two membrane-localized transcriptional activators on a single promoter. Mol. Microbiol. 38, 67-84. doi: 10.1046/j.13652958.2000.02111.x

Krzywinski, M., Schein, J., Birol, I., Connors, J., Gascoyne, R., Horsman, D., et al. (2009). Circos: an information aesthetic for comparative genomics. Genome Res. 19, 1639-1645. doi: 10.1101/gr.092759.109

Lagesen, K., Hallin, P., Rødland, E. A., Staerfeldt, H.-H., Rognes, T., and Ussery, D. W. (2007). RNAmmer: consistent and rapid annotation of ribosomal RNA genes. Nucleic Acids Res. 35, 3100-3108. doi: 10.1093/nar/gkm160

Langille, M. G. I., and Brinkman, F. S. L. (2009). IslandViewer: an integrated interface for computational identification and visualization of genomic islands. Bioinformatics 25, 664-665. doi: 10.1093/bioinformatics/btp030

Langille, M. G. I., Hsiao, W. W. L., and Brinkman, F. S. L. (2008). Evaluation of genomic island predictors using a comparative genomics approach. BMC Bioinformatics 9:329. doi: 10.1186/1471-2105-9-329

Laslett, D., and Canback, B. (2004). ARAGORN, a program to detect tRNA genes and tmRNA genes in nucleotide sequences. Nucleic Acids Res. 32, 11-16. doi: 10.1093/nar/gkh152

Li, L., Rock, J. L., and Nelson, D. R. (2008). Identification and characterization of a repeat-in-toxin gene cluster in Vibrio anguillarum. Infect. Immun. 76, 2620-2632. doi: 10.1128/IAI.01308-07

Lowe, T., and Eddy, S. (1997). tRNAscan-SE: a program for improved detection of transfer RNA genes in genomic sequence. Nucleic Acids Res. 25, 955-964. doi: 10.1093/nar/25.5.0955

Lux, T. M., Lee, R., and Love, J. (2011). Complete genome sequence of a free-living Vibrio furnissii sp. nov. strain (NCTC 11218). J. Bacteriol. 193, 1487-1488. doi: 10.1128/JB.01512-10

Manning, P. (1997). The tcp gene cluster of Vibrio cholerae. Gene 192, 63-70. doi: 10.1016/S0378-1119(97)00036-X

Mathur, J., and Waldor, M. (2004). The Vibrio cholerae ToxR-regulated porin $\mathrm{OmpU}$ confers resistance to antimicrobial peptides. Infect. Immun. 72, 3577-3583. doi: 10.1128/IAI.72.6.3577-3583.2004

Matté, G. R., Matté, M. H., Sato, M. I. Z., Sanchez, P. S., Rivera, I. G., and Martins, M. T. (1994). Potentially pathogenic vibrios associated with mussels from a tropical region on the Atlantic coast of Brazil. J. Appl. Microbiol. 77, 281-287.

Meibom, K. L., Blokesch, M., Dolganov, N. A., Wu, C.-Y., and Schoolnik, G. K. (2005). Chitin induces natural competence in Vibrio cholerae. Science 310, 1824-1827. doi: 10.1126/science.1120096

Meibom, K. L., Li, X. B., Nielsen, A. T., Wu, C.-Y., Roseman, S., and Schoolnik, G. K. (2004). The Vibrio cholerae chitin utilization program. Proc. Natl. Acad. Sci. U.S.A. 101, 2524-2529. doi: 10.1073/pnas.0308707101 
Miller, V. L., Taylor, R. K., and Mekalanos, J. J. (1987). Cholera toxin transcriptional activator toxR is a transmembrane DNA binding protein. Cell 48, 271-279. doi: 10.1016/0092-8674(87)90430-2

Murphy, R. A., and Boyd, E. F. (2008). Three pathogenicity islands of Vibrio cholerae can excise from the chromosome and form circular intermediates. J. Bacteriol. 190, 636-647. doi: 10.1128/JB.00562-07

Philippe, H., and Douady, C. J. (2003). Horizontal gene transfer and phylogenetics. Curr. Opin. Microbiol. 6, 498-505. doi: 10.1016/j.mib.2003.09.008

Reith, M. E., Singh, R. K., Curtis, B., Boyd, J. M., Bouevitch, A., Kimball, J., et al. (2008). The genome of Aeromonas salmonicida subsp. salmonicida A449: insights into the evolution of a fish pathogen. BMC Genomics 9:427. doi: 10.1186/1471-2164-9-427

Richards, T. A., Soanes, D. M., Foster, P. G., Leonard, G., Thornton, C. R., and Talbot, N. J. (2009). Phylogenomic analysis demonstrates a pattern of rare and ancient horizontal gene transfer between plants and fungi. Plant Cell 21, 1897-1911. doi: 10.1105/tpc.109.065805

Rivera, I., Chun, J., Huq, A., Sack, R., and Colwell, R. (2001). Genotypes associated with virulence in environmental isolates of Vibrio cholerae. Appl. Environ. Microbiol. 67, 2421-2429. doi: 10.1128/AEM.67.6.2421-2429.2001

Rodkhum, C., Hirono, I., Stork, M., Di Lorenzo, M., Crosa, J. H., and Aoki, T. (2006). Putative virulence-related genes in Vibrio anguillarum identified by random genome sequencing. J. Fish Dis. 29, 157-166. doi: 10.1111/j.13652761.2006.00692.x

Satchell, K. J. F. (2011). Structure and function of MARTX toxins and other large repetitive RTX proteins. Annu. Rev. Microbiol. 65, 71-90. doi: 10.1146/annurevmicro-090110-102943

Schmidt, H., and Hensel, M. (2004). Pathogenicity islands in bacterial pathogenesis. Clin. Microbiol. Rev. 17, 14-56. doi: 10.1128/CMR.17.1.14-56.2004

Schoolnik, G., and Yildiz, F. (2000). The complete genome sequence of Vibrio cholerae: a tale of two chromosomes and of two lifestyles. Genome Biol. 1, REVIEWS1016. doi: 10.1186/gb-2000-1-3-reviews1016

Scrudato, Lo., M., and Blokesch, M. (2012). The Regulatory network of natural competence and transformation of Vibrio cholerae. PLoS Genet. 8:e1002778. doi: 10.1371/journal.pgen. 1002778

Silva, A. J., Leitch, G. J., Camilli, A., and Benitez, J. A. (2006). Contribution of hemagglutinin/protease and motility to the pathogenesis of El Tor biotype cholera. Infect. Immun. 74, 2072-2079. doi: 10.1128/IAI.74.4.2072-2079.2006

Talavera, G., and Castresana, J. (2007). Improvement of phylogenies after removing divergent and ambiguously aligned blocks from protein sequence alignments. Syst. Biol. 56, 564-577. doi: 10.1080/10635150701472164

Tatusov, R., Galperin, M., Natale, D., and Koonin, E. (2000). The COG database: a tool for genome-scale analysis of protein functions and evolution. Nucleic Acids Res. 28, 33-36. doi: 10.1093/nar/28.1.33

Tettelin, H., Riley, D., Cattuto, C., and Medini, D. (2008). Comparative genomics: the bacterial pan-genome. Curr. Opin. Microbiol. 11, 472-477. doi: 10.1016/j.mib.2008.09.006
Thompson, F. L., Iida, T., and Swings, J. (2004). Biodiversity of vibrios. Microbiol. Mol. Biol. Rev. 68, 403-431. doi: 10.1128/MMBR.68.3.403-431.2004

Tsou, A., Cai, T., Liu, Z., Zhu, J., and Kulkarni, R. (2009). Regulatory targets of quorum sensing in Vibrio cholerae: evidence for two distinct HapR-binding motifs. Nucleic Acids Res. 37, 2747-2756. doi: 10.1093/nar/gkp121

Tsou, A., Liu, Z., Cai, T., and Zhu, J. (2011). The VarS/VarA two-component system modulates the activity of the Vibrio cholerae quorum sensing transcriptional regulator HapR. Microbiology 157, 1620-1628. doi: 10.1099/mic.0. 046235-0

Waack, S., Keller, O., Asper, R., Brodag, T., Damm, C., Fricke, W. F., et al. (2006). Score-based prediction of genomic islands in prokaryotic genomes using hidden Markov models. BMC Bioinformatics 7:142. doi: 10.1186/14712105-7-142

Wu, T.-K., Wang, Y.-K., Chen, Y.-C., Feng, J.-M., Liu, Y.-H., and Wang, T.-Y. (2007). Identification of a Vibrio furnissii oligopeptide permease and characterization of its in vitro hemolytic activity. J. Bacteriol. 189, 8215-8223. doi: 10.1128/JB.01039-07

Yamamoto, S., Izumiya, H., Mitobe, J., Morita, M., Arakawa, E., Ohnishi, M., et al. (2011). Identification of a chitin-induced small RNA that regulates translation of the tfoX gene, encoding a positive regulator of natural competence in Vibrio cholerae. J. Bacteriol. 193, 1953-1965. doi: 10.1128/JB. 01340-10

Yu, C., Lee, A., Bassler, B., and Roseman, S. (1991). Chitin utilization by marine bacteria. A physiological function for bacterial adhesion to immobilized carbohydrates. J. Biol. Chem. 266, 24260-24267.

Yu, C., Lee, A. M., and Roseman, S. (1987). The sugar-specific adhesion/deadhesion apparatus of the marine bacterium Vibrio furnissii is a sensorium that continuously monitors nutrient levels in the environment. Biochem. Biophys. Res. Commun. 149, 86-92. doi: 10.1016/0006-291X(87)91608-1

Conflict of Interest Statement: The authors declare that the research was conducted in the absence of any commercial or financial relationships that could be construed as a potential conflict of interest.

Received: 09 April 2014; accepted: 31 July 2014; published online: 21 August 2014. Citation: Lux TM, Lee R and Love J (2014) Genome-wide phylogenetic analysis of the pathogenic potential of Vibrio furnissii. Front. Microbiol. 5:435. doi: 10.3389/fmicb. 2014.00435

This article was submitted to Aquatic Microbiology, a section of the journal Frontiers in Microbiology.

Copyright (C) 2014 Lux, Lee and Love. This is an open-access article distributed under the terms of the Creative Commons Attribution License (CC BY). The use, distribution or reproduction in other forums is permitted, provided the original author(s) or licensor are credited and that the original publication in this journal is cited, in accordance with accepted academic practice. No use, distribution or reproduction is permitted which does not comply with these terms. 